\title{
THE AXISYMMETRIC PULSAR MAGNETOSPHERE: A CLASSICAL MODEL
}

\author{
R. Fitzpatrick and L. Mestel \\ Astronomy Centre, University of Sussex
}

The aim of this work (Fitzpatrick and Mestel 1988a, Fitzpatrick and Mestel 1988b) is to elucidate how a neutron star with a dipolar magnetic field of axis $\boldsymbol{k}$ and an instantaneous angular velocity $\alpha \boldsymbol{k}$ would spin down, within the framework of classical physics. The rotation of the highly conducting stellar crust generates differences of electric potential between field lines. An electron current flows from points of lower to higher potential via a dissipation domain located largely beyond the light-cylinder. Electrons leave the star's polar regions as a subrelativistic stream, flowing nearly along the lines of the poloidal field $B_{\mathrm{p}}$, picking up energy from the electric force and angular momentum from the moment about $k$ of the magnetic force. When the particles are sufficiently energetic they radiate, incoherently or coherently; the associated radiation of angular momentum enables them to drift across the lines of $B_{\mathrm{p}}$ under the essentially quadrupolar electric field $E$, ultimately to complete their circuits back to the star near the edge of the nolar cap.

A crucial feature of the dissipation domain is the beaming in the direction of the instantaneous velocity $v$ of the radiation from a relativistic particle, so that associated with a radiated power $\mathbb{P}$ per particle there is a momentum loss yielding a drag $-\left(\mathbb{P} / c^{2}\right) v$, and in particular an angular momentum loss rate $\left(\Omega \tilde{\omega}^{2} / c^{2}\right) \mathbb{P}$, where $\Omega(\dot{\omega}, z)$ is the angular velocity about $k$ of an electron at the point $P$ with cylindrical polar coordinates $(\tilde{\omega}, \varphi, z)$. The energy and angular momentum equations combine into

$v \cdot \nabla\left[\gamma m c^{2}\left(1-\frac{\alpha \Omega \tilde{\omega}^{2}}{c^{2}}\right)-e \psi\right]=-\mathbb{P}\left(1-\frac{\alpha \Omega \tilde{\omega}^{2}}{c^{2}}\right)$

where $\psi$ is the "non-corotational potential"-the difference in potential between $P$ and the point where the field line through $P$ leaves the star. The "centrifugal slingshot" term $\gamma m c^{2}\left(\alpha \Omega \tilde{\omega}^{2} / c^{2}\right)$ comes from the energy picked up from the "corotational potential" as the electron performs the trans-field "inertial drift" associated with its gain of angular momentum. Likewise, in the dissipation domain the drag force due to the loss of angular momentum accompanying the radiated power $\mathbb{P}$ leads to an electron drift onto a field line with a higher corotational potential and so to a gain of kinetic energy at the rate $\left(\alpha \Omega \bar{\omega}^{2} / c^{2}\right) \mathbb{P}$.

In the highly conducting, rigidly rotating stel- lar crust, $\psi$ is a constant. Integration of eq.(1) around an individual trajectory from the emission point to the return point then shows that most of the dissipation must occur in regions where $(\Omega \tilde{\omega} / c)(\alpha \tilde{\omega} / c)>1$, i.e. beyond the light-cylinder. The integral condition (Gold 1978, Holloway 1977) constraining the energy and angular momentum losses from the star is thus generalized to yield a "Gold-Holloway condition" for each trajectory.

Within the dissipation domain the velocity vector is fixed by the near balance of the Lorentz force and the radiation drag. As long as $E<B$, the poloidal velocity $v_{\mathrm{p}}$ remains approximately parallel to $B_{\mathrm{p}}$. The poloidal electron currents maintain a toroidal field $B_{\mathrm{t}}$, and in all the examples studied, $E<\left(B_{\mathrm{p}}^{2}+B_{\mathrm{t}}^{2}\right)^{1 / 2}$-and so $v_{\mathrm{p}}$ is nearly along $B_{\mathrm{p}}-$ until the electrons beyond the light-cylinder reach a sheet domain at the equator in which most of the trans- $B_{p}$ flow back towards the star occurs. A working model must have zero net charge on the star and magnetosphere with the dominant electric field at infinity quadrupolar. To ensure that $E>B$ on the equator at large distances, the magnetospheric toroidal currents must therefore have a dipole moment equal and opposite to that of the star, setting a strong constraint on the allowed model parameters.

The total power of the model is $\simeq B_{\mathrm{s}}^{2} r_{\mathrm{s}}^{6} \alpha^{4} / c^{3}$ (where $r_{\mathrm{s}}$ is the stellar radius and $B_{\mathrm{s}}$ the field strength at the stellar pole), of the same order as from the aligned star in the original GoldreichJulian picture and from the highly oblique DeutschPacini vacuum rotator. The typical $\gamma$-values reached by the radiating particles-and so also the frequency of the emitted radiation-depends on the dominant radiation process. Incoherent radiation of gamma-ray photons with an associated drag of the order of the Lorentz force occurs when $\gamma \simeq 2 \times 10^{7} / P_{1}^{7 / 4}$ (where $P_{1}=2 \pi / \alpha=$ pulsar rotation period in seconds and $B_{\mathrm{s}} \sim 10^{12} \mathrm{G}$ ). Particles of this energy can be easily produced from the potential differences on the star's surface in short period pulsars $\left(P_{1} \ll 1 s\right)$ such as Crab and Vela. Coherent radiation from cylindrical macroscopic blobs containing $N$ particles yields a drag per particle $\simeq N e^{2} \gamma^{4} \alpha^{2} / c^{2}$ (Schiff 1946, Goldreich and Keeley 1971), and this can balance the Lorentz force on a particle at much smaller values of $\gamma$. A rough estimate suggests that the energy (electrostatic plus 
kinetic) of the dissipation domain will be minimized if spontaneous clumping occurs into such blobs with each containing $N \simeq m^{2} c^{4} / e^{3} B_{\mathrm{LC}}$ particles and with the required $\gamma \simeq\left(e B_{\mathrm{LC}} / m c \alpha\right)^{1 / 2} \simeq 4 \times 10^{3} / P_{1}$ where $B_{\mathrm{LC}}$ is a typical value of $B_{\mathrm{p}}$ near the lightcylinder. The emission would then be in the radio band.

To validate the model requires the construction of mutually self-consistent electromagnetic fields and density-velocity fields of both the circulating electrons and the corotating or sub-rotating electrons and positive charges in their different domains. Some limited progress towards this goal is reported in (Fitzpatrick and Mestel 1988a, Fitzpatrick and Mestel 1988b). Further work will concentrate rather on developments suggested by the model itself. A difficulty for models which involve large potential gradients along $\boldsymbol{B}_{\mathrm{p}}$-and so acceleration to high $\gamma$-values near the star-is that the consequent pair production will act to short out the electric field along $B_{\mathrm{p}}$ as soon as the $e^{ \pm}$density is of the order of the Goldreich-Julian density. In the present model, the electrons returning to the star have tapped all the energy available by the crossing of field lines beyond the light-cylinder, enforced by the radiation of angular momentum; they may, therefore, be expected to return to the star with high $\gamma$-values and so to generate a mixed plasma via pair production in the high $B$ field near the star, or more effectively by surface spallation (Rylov 1977, Fitzpatrick 1988). One is then led to a picture in which circulation of the primary electrons acts as a trigger for the continuous generation of a dense mixed magnetosphere. Once the plasma kinetic energy density near the light-cylinder exceeds locally the magnetic energy density, a plasma wind will flow to infinity, carrying off energy of the same order as that lost by radiation. 